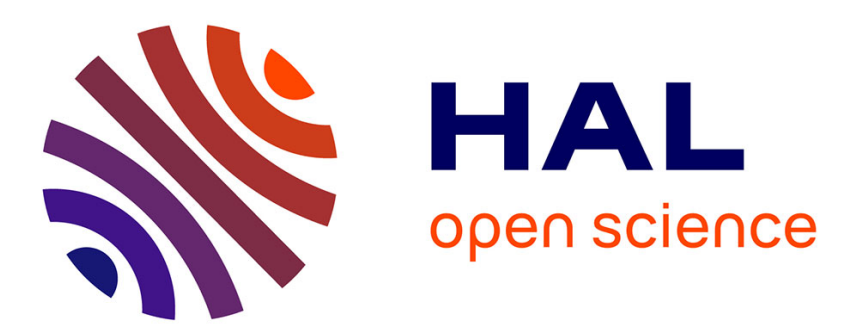

\title{
Adsorption Deformation and Structural Transitions in Metal-Organic Frameworks: From the Unit Cell to the Crystal
}

François-Xavier Coudert, Anne Boutin, Alain H. Fuchs, Alexander V. Neimark

\section{- To cite this version:}

François-Xavier Coudert, Anne Boutin, Alain H. Fuchs, Alexander V. Neimark. Adsorption Deformation and Structural Transitions in Metal-Organic Frameworks: From the Unit Cell to the Crystal. Journal of Physical Chemistry Letters, 2013, 4 (19), pp.3198-3205. 10.1021/jz4013849 . hal-02116926

\section{HAL Id: hal-02116926 \\ https://hal.science/hal-02116926}

Submitted on 1 May 2019

HAL is a multi-disciplinary open access archive for the deposit and dissemination of scientific research documents, whether they are published or not. The documents may come from teaching and research institutions in France or abroad, or from public or private research centers.
L'archive ouverte pluridisciplinaire HAL, est destinée au dépôt et à la diffusion de documents scientifiques de niveau recherche, publiés ou non, émanant des établissements d'enseignement et de recherche français ou étrangers, des laboratoires publics ou privés. 


\section{Adsorption Deformation and Structural Transitions}

\section{in Metal-Organic Frameworks: From the Unit Cell \\ to the Crystal}

François-Xavier Coudert, ${ }^{a *}$ Anne Boutin ${ }^{b}$ Alain H. Fuchs,${ }^{a}$ and Alexander V. Neimark ${ }^{\circ \cdot}$

- CNRS and Chimie ParisTech, 11 rue Pierre et Marie Curie, 75005 Paris, France

' Département de Chimie, École Normale Supérieure, CNRS-ENS-UPMC, 24 rue Lhomond, 75005 Paris, France

- Department of Chemical and Biochemical Engineering, Rutgers University, New Jersey 08854, USA

\section{Corresponding Author}

* fx.coudert@chimie-paristech.fr (F.-X.C.); aneimark@rutgers.edu (A. V. N.) 
ABSTRACT. Much attention has recently been focused on Soft Porous Crystals, a fascinating subclass of Metal-Organic Frameworks that behave in a remarkable stimuli-responsive fashion, presenting structural changes of large amplitude in response to guest adsorption, mechanical pressure or variations in temperature. In this perspective, we summarize the recently developed thermodynamic and mechanical theoretical models developed for the understanding of these materials, based on the simple concepts of adsorption stress and the osmotic thermodynamic ensemble. We show how these models aim at providing a coherent picture of adsorption-induced deformation and structural transitions in flexible Metal-Organic Frameworks, all the way from the length scale of the unit cell to that of the full crystal. In particular, we highlight the new perspectives opened by these models, as well as some of the important open questions in the field.

\section{TOC GRAPHICS}

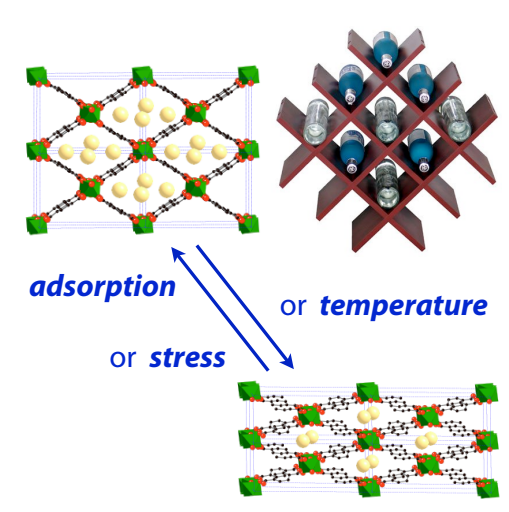

KEYWORDS: adsorption, adsorption stress, structural transitions, porous materials, breathing, metal-organic frameworks. 
Much attention has recently been focused on metal-organic frameworks (MOFs), a wide class of designer microporous materials that garner a lot of interest for their potential applications in the fields of separation, catalysis, strategic gas capture and storage, and drug delivery.12 Soft Porous Crystals (SPCs) represent a specific subclass of MOFs with remarkable flexibility. These materials feature dynamic crystalline frameworks with reversible structural transformations of large amplitude in response to variation of external physical stimuli such as gas adsorption, temperature, or mechanical pressure. ${ }^{3}$ SPCs display a large gamut of flexibility mechanisms depicted in Figure 1,4 including: local framework dynamics, as sorption-induced linker reorientation in ZIF-8; negative thermal expansion, as in the IRMOF family of materials; gradual swelling upon uptake of solvent molecules, as demonstrated in the MIL-88 family; gate opening, which involves an abrupt structural transition between close and open pore structures that is induced by gas adsorption (e.g. in the ELM family); ${ }^{:}$and the breathing phenomenon, which consists of two successive adsorption-induced crystal-to-crystal transformations, for example in the case of the MIL-53 family of materials, from a large pore (lp) state to a narrow pore (np) state, and back again to the lp state.' A growing number of SPC structures were synthesized and reported in the literature, and although none of them have yet been directly used at the industrial scale, ${ }^{10}$ they have been proposed for a large range of potential practical applications. In addition to the general application of MOFs, flexible frameworks are expected to present an intrinsic interest due to their large-scale stimuli-responsive transformations," which could leverage novel nano-biotechnologies, such as sensing for detecting traces of organic molecules,,$^{12}$ slow release of drugs for long-release single-injection therapies,,$^{13}$ and specific gas separations..$^{14.15}$ 
The adsorption-induced deformation and adsorption-induced transitions in flexible MOFs were first addressed in the literature with respect to their structures and energetics, both experimentally and using molecular simulation methods. From the theoretical point of view, there are two key fundamental concepts for understanding the behaviors reported in the literature. First, the adsorption-induced deformation can be viewed as a response of the porous material to an adsorption-induced stress, exerted by the adsorbed fluid on the host solid. ${ }^{16}$ Secondly, a rigorous thermodynamic description of the interplay between adsorption and deformation including structural transitions is provided by the use of the osmotic ensemble, ${ }^{17}$ a semi-open statistico-mechanical ensemble in which the quantity of solid, temperature and mechanical stress are fixed, while the quantity of guest molecules and the unit cell shape and volume may change. The osmotic ensemble has been used to build theoretical models of the influence of adsorption on the equilibrium between metastable host structures. ${ }^{4}$ Such analytical models are useful in rationalizing the various types of structural transitions observed and the key parameters controlling their occurrence, ${ }^{17}$ in processing the experimental data, ${ }^{18}$ or in making predictions of mixture co-adsorption from pure component data. ${ }^{19}$

The osmotic ensemble has also been used as a basis for designing molecular simulation methods focused on adsorption in flexible materials. ${ }^{2021,2223}$ Atomistic molecular simulations enable one to link microscopic details of the host structure and host-guest interactions to macroscopic behavior with an unmatched degree of accuracy. However, they have limitations when it comes to understanding the mechanisms of deformation and structural transitions. One limitation is the length and time scales currently achievable, directly due to the computational cost of the atomistic description: most atomistic simulations range in size between one and a few dozen unit cells, which is far from the actual size of a micrometric (or even submicrometric) crystal. The 
second limitation is that molecular simulation of porous materials typically use periodic boundary conditions, in order to lower the computational cost and to minimize the finite size and boundary effects, but the periodic boundary conditions do not reproduce the conditions of the sample boundaries and impose artificial constraints on the deformation pathways in the material. This approximation is thus no longer valid for understanding the mechanisms of deformation and structural transitions, and more adequate simulation techniques have to be developed to avoid this issue.

Thus, while adsorption-induced deformation and structural transitions are by now well understood from the point of view of thermodynamics and energetics, a mechanical approach to the dynamics of these phenomena has not been developed. In particular, there have been so far no experimental studies of structural transition dynamics at the scale of the monocrystal. These aspects are, however, of large importance. Firstly, experimental data show that structural transitions in SPC are almost always associated with wide hysteresis loops, whether they are triggered by adsorption, temperature, or mechanical pressure. Secondly, recent experimental results from the Kitagawa group clearly show that the crystal size is a critical factor in structural transitions upon adsorption. ${ }^{24}$ Thus, it is crucial to understand the dynamics of structural transitions at the crystal scale, both from the fundamental point of view and for practical applications of these materials.

By relying upon the fundamental concepts of adsorption stress and osmotic ensemble outlined above, we proposed the first mechanical approach to the adsorption-induced structural transitions, the so-called stress model. ${ }^{25}$ We suggested that adsorption-induced stress exerted on the material acts as a stimulus that triggers structural transitions of the framework, which occur when the stress reaches a certain critical threshold. Based on the stress model, we provided a 
quantitative description of the double breathing transitions in MIL-53(Al) upon $\mathrm{CO}_{2}, \mathrm{Xe}$, and $\mathrm{CH}_{4}$ adsorption. ${ }^{26}$ Shortly after that, Beurroies et al..$^{27}$ demonstrated that the structural transitions in MIL-53(Cr) could be also set off by uniform mechanical compression of the powder immersed in liquid mercury, as shown in Figure 2. We used this data and as well as the adsorption data and in situ X-ray diffraction patterns reported for $\mathrm{CO}_{2}$ adsorption on MIL-53(Cr) to show that both adsorption and mechanical compression mechanisms of framework transformations could be described within a unified thermodynamic model based on the minimization of the osmotic thermodynamic potential. ${ }^{28}$ In particular, we noted that the magnitude of the adsorption stress exerted inside the pores by guest molecules, which is required for inducing the breathing transition, corresponded to the magnitude of the external mercury pressure applied from the outside.

The thermodynamic analysis of equilibrium states within the osmotic ensemble and the adsorption stress model provides a general framework for the description of the structural transition (pore opening, gating, breathing) as a path followed by the system along the free energy landscape from one minimum to another over a barrier, which is crossed upon achieving the threshold stress. The equilibrium transition conditions and respective phase diagrams, as well as the energy barrier can be estimated on the level of single cells by using different simulation approaches. ${ }^{22.9930}$ However, this thermodynamic treatment does not yield any information into the local elastic deformation of the material, nor on the pathways and dynamics of structural transformations.

Most of the earlier studies were focused on key scalar properties, some of them isotropic (bulk modulus), some of them specific to key crystallographic axes (Young's modulus, hardness). ${ }^{31}$ Recently, Tan et al. ${ }^{32}$ have demonstrated on the case of ZIF-8 that state-of-the-art experimental 
techniques and theoretical calculations can be used to compute the full tensor of second-order elastic constants of MOFs, thus fully accounting for the anisotropy of framework elastic properties. Based on either quantum chemistry calculations or classical molecular dynamics, the tensorial analysis of elastic properties reveals a great deal of information about flexible MOFs. By using $a b$ initio calculations of the elastic tensor, we demonstrated that high anisotropy and distinct directions of anomalously low Young and shear moduli are the key signatures of "breathing" MOFs, ${ }^{33}$ in particular those of a wine-rack framework topology, such as the MIL-53 family. The anisotropy can reach a 400:1 ratio between the most rigid and weakest directions. Comparison between the characteristic 3D maps of elastic moduli for MIL-47 and MOF-5 structures are shown in Figure 3. While the ratio of the elastic moduli in "soft" and "hard" directions is $\sim 100$ for flexible MIL-47, it is only $\sim 2$ for non-compliant MOF-5. Further, we showed that the application of hydrostatic pressure on ZIF-8 leads to a shear softening and subsequent mechanical instability, which may cause crystal amorphization. ${ }^{35}$ The characteristic dependences of the unit cell parameter and elastic constants on the confining pressure for ZIF-8 are presented in Figure 4. Another factor that should be taken into account while analyzing the adsorption induced deformation is the influence of the adsorbate of the crystal elastic moduli, which was earlier demonstrated by Coasne et al. drawing on the example of silicalite zeolites. ${ }^{36}$ We established this effect in MOFs in the case of the ZIF-8 framework. ${ }^{35}$

The detailed modeling of the anisotropic elastic response on the level of single cell provides important information about the deformation pathways and helps identify key order parameters of deformation and structural transitions, which define the framework geometry at given thermodynamic conditions. The next step is to link this microscopic information on mechanical properties to a mechanism of transitions at the level of multiple unit cells. This can be done, at 
least in a first approximation, by using simple geometric models of the unit cell (depending on its crystal class) with one or few variable order parameters, and writing down the generic equations for elastic compatibility conditions.

In the case of MIL-53(Al or Ga), for example, the analysis of the unit cell elastic tensor ${ }^{33}$ confirmed that the softest mode of deformation is the cell shear perpendicular to the channel direction. As such, the cell deformation is characterized by one primary order parameter related to the volumetric strain, or to the angle of the pore channel rhombus cross-section, which varies from $\sim 79^{\circ}$ in lp states to $\sim 40^{\circ}$ in np states (Figure 5, left). In order to couple the deformations of the cells within the 3D framework, we invoked the general theory of elasticity and showed that due to the Saint-Venant elastic compatibility conditions, the structural transition between the $\mathrm{lp}$ and np phases happens collectively by shearing of the 2D layer of unit cells, as schematized in Figure 5, right. ${ }^{37}$ Based on this conclusion, we built the simplest possible, or minimalistic physical model of breathing transitions at the scale of the crystal, including only the most essential elements: host elastic terms (parabolic local term reflecting linear elasticity regime, and elastic coupling between neighboring cells), free energy difference and free energy barrier between the lp and np phases, as well as host-guest coupling in the form of adsorption energy and adsorption stress.$^{38}$ Monte Carlo simulation of coupled adsorption and deformation on the crystal level performed with this minimalistic model allowed us to reveal the origin of the hysteresis arising in adsorption-induced structural transitions, and to gain an insight into the effect of crystal size on macroscopic behavior. We showed a possibility of the phase coexistence within one crystal in the transition region and the dynamic nature of this coexistence. In particular, we found that depending on the system parameters the lp-np transition may occur either reversibly or with a prominent hysteresis, and the cell layers of lp and np phases may be 
distributed in the crystal either randomly or forming just a few clusters, which rapidly grow in time and coalesce, see Figure 6. Work is now going on to extend this simple qualitative model into a quantitative lattice-based modeling method, by using as input the mechanical and adsorption properties at the microscopic scale, determined either from experiments or from atomistic level molecular simulation (adsorption energy, adsorption strain, elastic moduli). This kind of multiscale approach is fundamental to the understanding, bridging the cell level models and macroscopic dynamics of the crystal structure transformation.

The concepts of the osmotic thermodynamic ensemble and the adsorption stress have found already multiple applications in modeling adsorption deformation phenomena, some of which follow directly the models outlined above and extend them, ${ }^{3940}$ and some of which offer similar or alternative models based on the same basic concepts. In particular, Miyahara and co-workers in a series of papers ${ }^{30,4,1,42}$ used free energy calculation methods to study the gate opening behavior in interpenetrated frameworks of "jungle gym" of cubic lattice structure (model systems to interpenetrated and interdigitated coordination polymers with cubic lattice $\left.{ }^{43}\right)$, and the gate opening and further swelling in stacked-layer pillared structures (which represent Elastic Layerstructured Metal organic-frameworks, or ELMs $\left.{ }^{44}\right)$. Pera-Titus and Farrusseng proposed a formulation of thermodynamic isotherms for describing S-shaped adsorption curves in the case of adsorption-induced structural transitions, as well as a way of estimating phase transition energies. ${ }^{45}$ The group of Maurin has used hybrid Molecular Dynamics/Monte Carlo simulations in the osmotic ensemble to study the breathing of materials of the MIL-53 and MIL-47 families, first in presence of adsorbates,$^{46,47}$ then with variations in mechanical pressure and temperature.$^{48,49}$ Very recently, Zhang et al..$^{50}$ used a similar methodology to simulate $\mathrm{N}_{2}$ adsorption isotherm in ZIF-8, while accounting for the crucial local framework flexibility of the material. Zang et al.s1 
applied the osmotic ensemble to study swelling of single-walled aluminosilicate nanotube bundles and cesium montmorillonite. Santander et al.52 performed Monte Carlo simulations in the osmotic ensemble to investigate the expansion of silicalite upon adsorption of biomass-derived solutions. Brochard et al..$^{53}$ attempted to augment the classical Biot poromechanics with the adsorption-induced stress in the case of isotropic deformation. Kowalczyk et al ${ }^{54}$ determined the pore size dependence of the adsorption stress in microporous carbons. Yang et al ${ }^{55,56}$ studied the effects of pore swelling and contraction in coal during carbon dioxide and methane adsorption. Gor and Neimark ${ }^{5,58}$ explained the non-monotonic deformation of mesoporous adsorbents by calculating the adsorption stress resulting from the competition between the disjoining and capillary pressures. Bousquet et al..$^{29}$ constructed a general model for analyses of metastability of intermediate structures during adsorption-induced transitions in SPC.

While the coupled thermodynamic and mechanical approach to adsorption-induced deformation and structural transitions in SPC has brought about new physical insight into these phenomena, there are still a number of important open questions that remain to be addressed. In the following, we discuss what we consider as some of the most topical and challenging unresolved problems in this field of research. One of the much-discussed issues is the possibility of phase coexistence within one crystal. Although our minimalistic model, described above, indicates that phase coexistence could occur at certain conditions inside a single crystal, it is not clear under what conditions of time and length scales it may happen in real systems. While in situ X-ray diffraction data ${ }^{9}$ clearly indicates the coexistence of different phases in polycrystalline samples, no experimental evidence that we are aware of can confirm (or refute) that phase coexistence is possible at the single crystal level, and it seems difficult to design a relevant experiment (to give one example, most MOFs are unstable under electron irradiation at SEM 
conditions). However, such information could be obtained with in silico experiments, using molecular simulations of a system of sufficiently large scale. One of the great upcoming challenges for molecular simulation is the direct modeling of mechanical properties and stability of deformable structures at this scale, ranging from reversible and hysteretic transformations to irreversible formation of dislocations and fractures. Multiscale approaches such as those described in ref. 37 will be crucial for this, allowing to bridge length and time scales, while existing work on multiscale MOF modeling has until now been mostly limited to the creation of force fields from first principles reference data..$^{59,60,61}$

Another series of open questions is spawned by the recent observation of the very high anisotropy of SPC's elastic properties..$^{33}$ This anisotropy, which reaches up to two orders of magnitude between the stiffest and softest deformation modes, hints that a scalar treatment of stress and strain, as currently done in both thermo-mechanical models and classical poromechanics, ${ }^{62,53}$ is likely valid only as a first-order approximation. Further extensions of poromechanics models require a more detailed accounting of the system anisotropy, for example by the introduction of additional (secondary) order parameters that would complement the currently used primary order parameter, which is the volumetric strain. Moreover, a connection needs to be established between the microscopic anisotropy of the system and the overall symmetry of the macroscopic sample. During the transition from the microscopic to the macroscopic scale, the anisotropy is averaged out, and this needs to be accounted for within the mechanical model. The proper upscaling is critically important: while all experimental and theoretical studies of SPCs are done on the level of micrometer-sized crystals, the potential practical applications of these materials will necessarily involve composite materials in the form of films or particles, where anisotropic MOF crystals are glued together by a binder into 
macroscopically isotropic samples. One example of such process would be the pelletization of MOF powder to produce adsorbents or catalysts. In such a composite system, the phase transformations in SPC's single crystals under action of an external stimulus (adsorbing gas or temperature) could be affected, or even suppressed, by particle-particle and particle-binder constraints. This brings about the confining stress acting on the crystal in addition to the adsorption stress. In order to consider deformation and phase transformation in confined crystals, it is necessary to marry the macroscopic poromechanics treatment at the level of the composite particle and the microscopic thermodynamic and mechanical approaches discussed in this Perspective.

It is worth noting in conclusion that the concepts highlighted and the methods described in this Perspective are not limited to SPCs and are fully applicable to other compliant porous solids. Phenomena of adsorption deformation and structural transitions in SPCs have attracted a lot of recent interest because of the large-scale stimuli-induced deformations, but flexibility is present in all nanoporous materials to a certain extent. Among the topical problems, where thermodynamic and mechanical models could be of great use, are deformation of micro- and mesoporous adsorbents and catalysts under technological conditions; swelling of clays and laminated minerals; shrinkage and swelling of coal and its influence on the secondary methane recovery and carbon dioxide sequestration; mechanical properties of low- $k$ dielectric films; hydrofracturing in shale-gas reservoirs; hydration-dependent mechanical properties of cement and other building materials; porous membranes and electrodes in fuel cells and batteries, etc. Although some attempts in these directions have been already made, a comprehensive poromechanics of nanostructured adsorbents still remains to be built. 


\section{Author Biographies}

François-Xavier Coudert is a CNRS researcher at Chimie ParisTech, a chemical engineering college in Paris, France. His research interests are in the area of molecular adsorption in nanoporous materials, with particular focus on developing theoretical models and tools, as well as molecular simulation algorithms, for the description of Soft Porous Crystals. See http://www.chimie-paristech.fr/molsim for further details.

Anne Boutin is CNRS research director at the École Normale Supérieure (ENS) in Paris, France. Her research interests cover the theory, modeling and molecular simulations of molecular fluids and ions adsorbed in porous solids. For more information see http://hartree.chimie.ens.fr/theorie/boutin/

Alain H. Fuchs is President of the French CNRS (Centre National de la Recherche Scientifique), Senior Professor at Université Pierre et Marie Curie (Paris, France) and Fellow of the Royal Society of Chemistry. His research interests cover the thermodynamics and statistical thermodynamics of confined systems and nanoporous materials (zeolites, MOFs, surface force systems, ...)

Alexander V. Neimark is a Distinguished Professor in Chemical and Biochemical Engineering at Rutgers University. His research involves studies of thermodynamic, transport, and structural properties of nanoscale systems, particularly nanoporous materials and selfassembled soft matter, by using statistical mechanics and multiscale molecular and coarsegrained simulations. See http://sol.rutgers.edu/ aneimark/ for more details. 

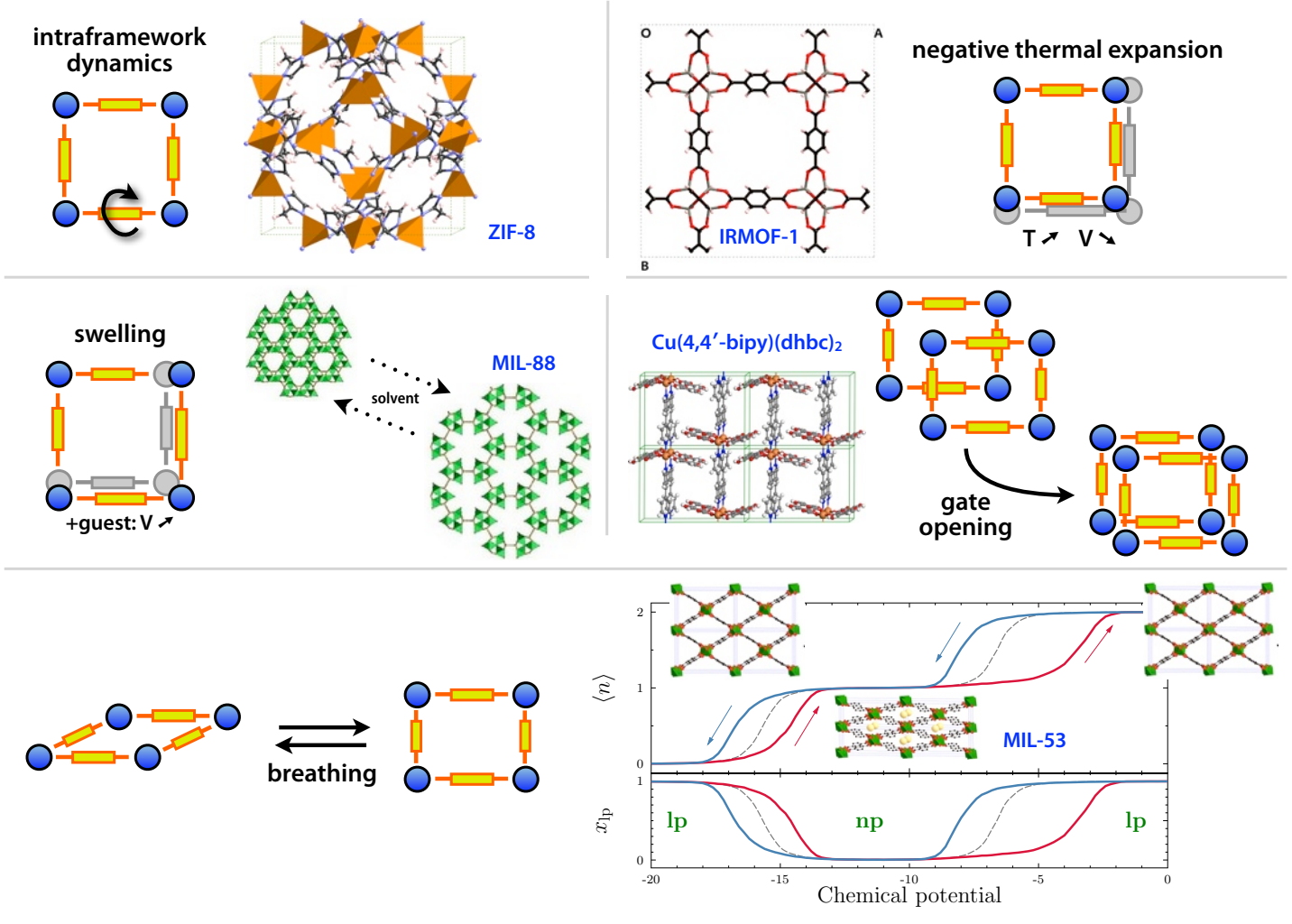

Figure 1. Different categories of dynamic metal-organic frameworks, each illustrated by a material displaying the phenomenon. The lower panel also depicts the typical hysteretic two-step adsorption-desorption isotherms of breathing materials (red and blue respectively, upper half), along with evolution of phase composition (lower half). (Modified from ref. 4) 

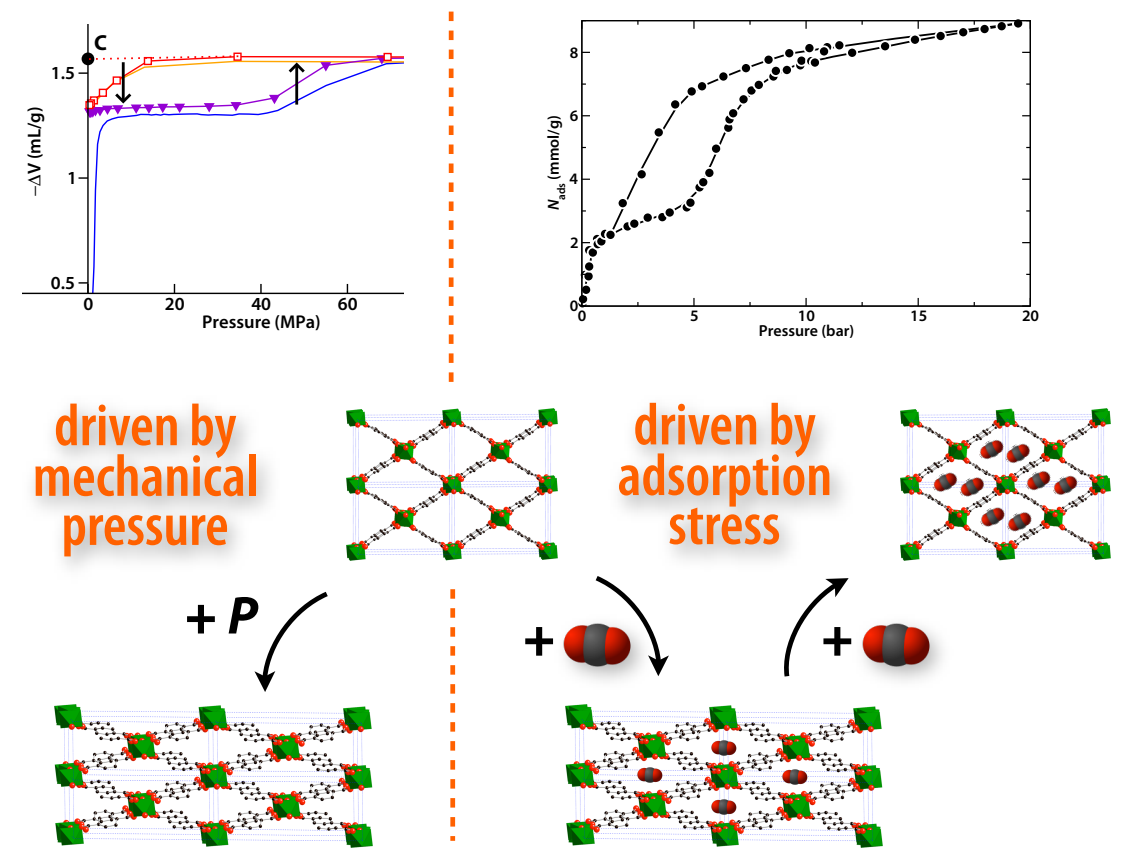

Figure 2. The large pore (lp) to narrow pore (np) structural transition in MIL-53 can be induced either by mechanical pressure or adsorption, as schematized in the lower half of the graphics. Top left: Intrusion-extrusion experiments in mercury, featuring the volume variation of the sample $(\Delta V)$ as a function of mechanical pressure; two clear steps are seen corresponding to lpnp and np-lp transitions (black arrows). Top right: $\mathrm{CO}_{2}$ adsorption and desorption isotherms at 304 K. (Redrawn from ref. 28) 


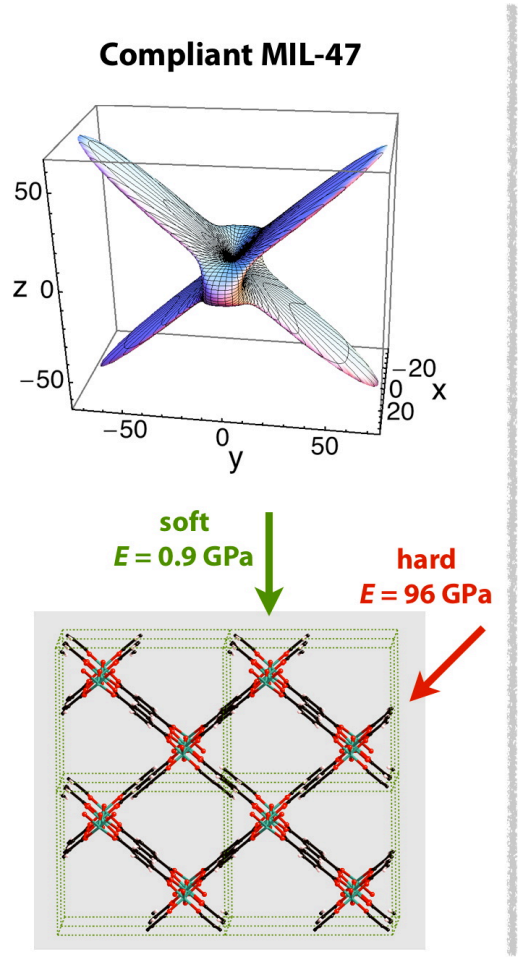

\section{Non-compliant MOF-5}
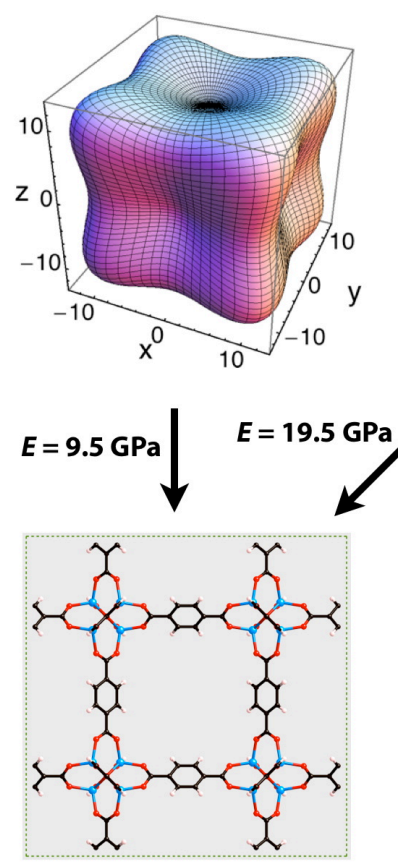

Figure 3. Comparison of the directional Young's modulus (3D representation, upper panels) of breathing MOF MIL-47 and non-breathing MOF-5. On the bottom panel are indicated the stiffest and softest directions of deformation (minimal and maximal Young's modulus), and the respective values of Young's modulus $E$. 

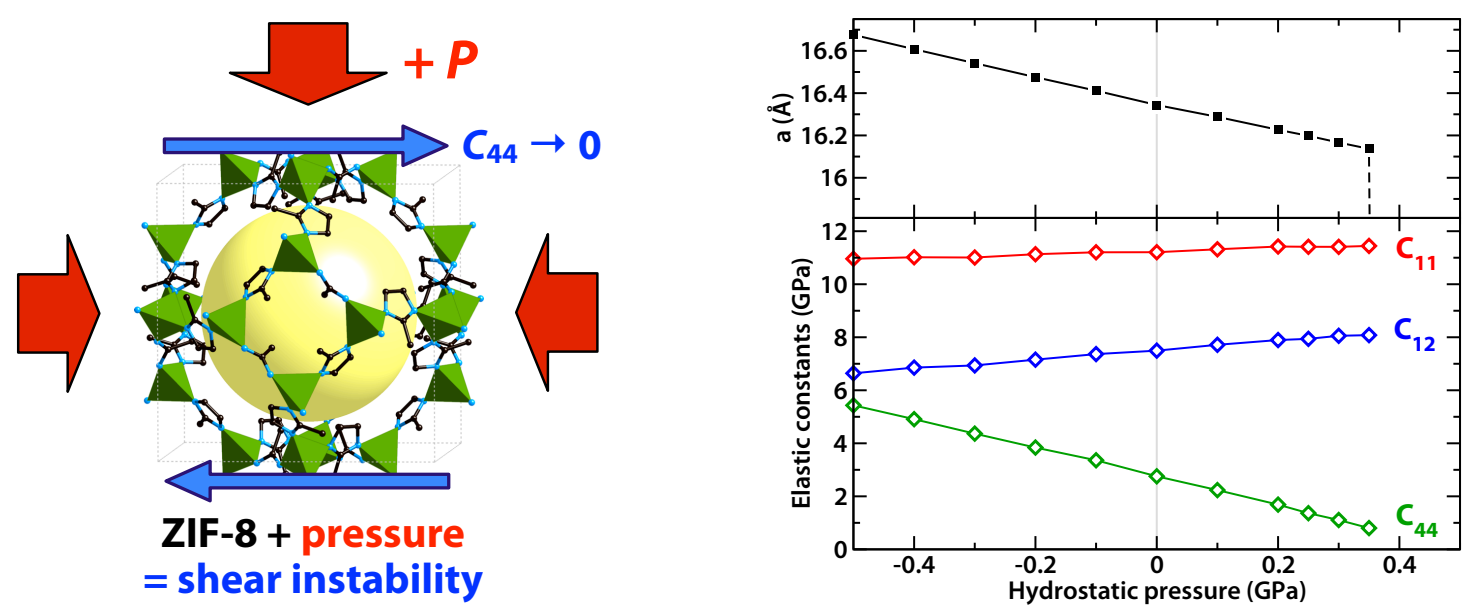

Figure 4. Unit cell parameter $a$ and elastic constants $C_{11}, C_{12}$, and $C_{44}$ of ZIF-8 as a function of hydrostatic pressure at $77 \mathrm{~K}$. (Reprinted from ref. 35)

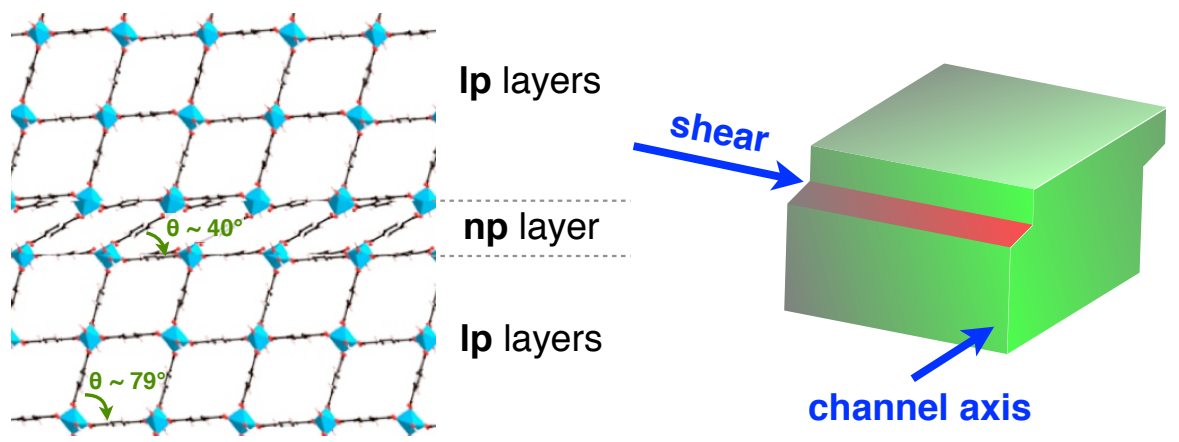

Figure 5. Schematic representation of the MIL-53 framework after the first event of lp-np transformation, which involves in-plane shear of a 2D layer of cells in a direction orthogonal to the channel axis. (Reprinted from ref. 37) 

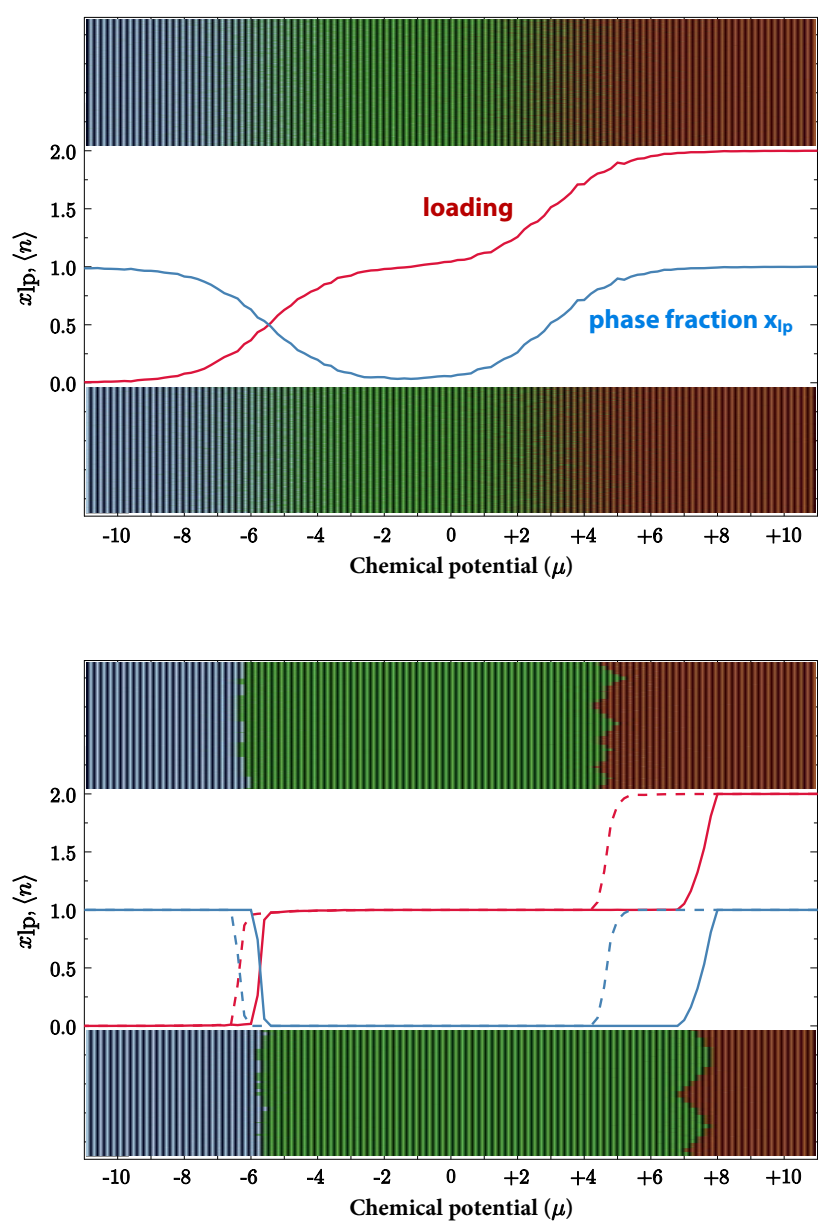

Figure 6. Adsorption isotherms, $\langle n\rangle$ (red curves); phase fraction, $x_{\mathfrak{1 p}}$ (blue curves) as a function of chemical potential $\mu$. On top and bottom are represented spatial distribution of phases and loadings, for adsorption (bottom) and desorption (top), at corresponding $\mu$. Each vertical bar represents the 1D succession of 2000 layers colored with respect to their states: blue - empty lp phase; grey - empty np phase; green - np phase with one particle; white - lp phase with one particle; red - lp phase with two particles; and black - np phase with two particles (not observed in these simulations). Upper and lower panel represent different sets of parameters from the model, showing the possibility for both reversible transitions and hysteresis (see ref. 38 for details). (Reprinted from ref. 38) 


\section{REFERENCE}

(1) Zhou, H. C.; Long, J. R.; Yaghi, O. M. Introduction to Metal-Organic Frameworks. Chem. Rev. 2012, 112, 673-674; and other papers of the Chem. Rev. special issue.

(2) Metal-Organic Frameworks: Applications from Catalysis to Gas Storage, Farrusseng, D., Ed.; Wiley-VCH, Weinheim, 2011.

(3) Horike, S.; Shimomura, S.; Kitagawa, S. Soft Porous Crystals. Nature Chemistry 2009, 1, $695-704$.

(4) Coudert, F.-X.; Boutin, A.; Jeffroy, M.; Mellot-Draznieks, C.; Fuchs, A. H. Thermodynamic Methods and Models to Study Flexible Metal-Organic Frameworks. ChemPhysChem 2011, 12, 247-258.

(5) Fairen-Jimenez, D.; Moggach, S. A.; Wharmby, M. T.; Wright, P. A.; Parsons, S.; Duren, T. Opening the Gate: Framework Flexibility in ZIF-8 Explored by Experiments and Simulations. J Am Chem Soc 2011, 133, 8900-8902.

(6) Dubbledam, D.; Walton, K. S.; Ellis, D. E.; Snuur, R. Q. Exceptional Negative Thermal Expansion in Isoreticular Metal-Organic Frameworks. Angew. Chem. Int. Ed. 2007, 46, 44964499.

(7) Serre, C.; Mellot-Draznieks, C.; Surblé, S.; Audebrand, N.; Filinchuk, Y.; Férey, G. Role of Solvent-Host Interactions That Lead to Very Large Swelling of Hybrid Frameworks. Science 2007, 315, 1828-1831. 
(8) Cheng, Y.; Kajiro, H.; Noguchi, H.; Kondo, A.; Ohba, T.; Hattori, Y.; Kaneko, K.; Kanoh, H. Tuning of Gate Opening of an Elastic Layered Structure MOF in CO2 Sorption with a Trace of Alcohol Molecules. Langmuir 2011, 27, 6905-6909.

(9) Serre, C.; Bourrelly, S.; Vimont, A.; Ramsahye, N. A.; Maurin, G.; Llewellyn, P. L.; Daturi, M.; Filinchuk, Y.; Leynaud, O.; Barnes, P.; Férey, G. An Explanation for the Very Large Breathing Effect of a Metal-Organic Framework during CO2 Adsorption. Adv. Mater. 2007, 19, 2246.

(10) Czaja, A. U.; Trukhan, N.; Müller, U. Industrial applications of metal-organic frameworks. Chem. Soc. Rev. 2009, 38, 1284-1293

(11) Bureekaew, S.; Shimomura, S.; Kitagawa, S. Chemistry and application of flexible porous coordination polymers. Sci. Technol. Adv. Mater. 2008, 9, 014108.

(12) Férey, G.; Serre, C. Hybrid porous solids: past, present, future. Chem. Soc. Rev. 2009, 38, 1380-1399.

(13) Horcajada, P.; Serre, C; Maurin, G.; Ramsahye, N. A., Balas, F.; Vallet- Regi, M.; Sebban, M; Taulelle, F.; Férey, G. Flexible Porous Metal-Organic Frameworks for a Controlled Drug Delivery. J. Am. Chem. Soc. 2008, 130, 6774.

(14) Thallapally, P. K.; Tian, J.; Kishan, M. R.; Fernandez, C. A.; Dalgamo, S. J.; McGrail, P. B.; Warren, J. E.; Atwood, J. L. Flexible (Breathing) Interpenetrated Metal-Organic Frameworks for CO2 Separation Applications. J. Am. Chem. Soc. 2008, 130, 16842-16843.

(15) Remy, T.; Baron, G. V.; Denayer, J. F. M. Modeling the Effect of Structural Changes during Dynamic Separation Processes on MOFs. Langmuir 2011, 27, 13064-13071. 
(16) Ravikovitch, P. I.; Neimark, A. V. Density Functional Theory Model of Adsorption Deformation. Langmuir 2006, 22, 10864-10868

(17) Coudert, F.-X.; Jeffroy, M.; Fuchs, A. H.; Boutin, A.; Mellot-Draznieks, C. Thermodynamics of Guest-Induced Structural Transitions in Hybrid Organic-Inorganic Frameworks. J. Am. Chem. Soc. 2008, 130, 14294-14302.

(18) Boutin, A.; Springuel-Huet, M.-A.; Nossov, A.; Gédéon, A.; Loiseau, T.; Volkringer, C.; Férey, G.; Coudert, F.-X.; Fuchs, A. H. Breathing Transitions in MIL-53(Al) Metal-Organic Framework Upon Xenon Adsorption. Angew. Chem. Int. Ed. 2009, 48, 8314 -8317.

(19) Coudert, F.-X. The osmotic framework adsorbed solution theory: predicting mixture coadsorption in flexible nanoporous materials. Phys. Chem. Chem. Phys. 2010, 12, 10904-10913.

(20) Ghoufi, A.; Maurin, G. Hybrid Monte Carlo Simulations Combined with a Phase Mixture Model to Predict the Structural Transitions of a Porous Metal-Organic Framework Material upon Adsorption of Guest Molecules. J. Phys. Chem. C 2010, 114, 6496-6502.

(21) Zang, J.; Nair, S.; Sholl, D. S. Osmotic ensemble methods for predicting adsorptioninduced structural transitions in nanoporous materials using molecular simulations. J. Chem. Phys. 2011, 134, 184103.

(22) Bousquet, D.; Coudert, F.-X.; Boutin, A. Free energy landscapes for the thermodynamic understanding of adsorption-induced deformations and structural transitions in porous materials. J. Chem. Phys. 2012, 137, 044118. 
(23) Santander, J. E.; Tsapatsis, M.; Auerbach, S. M. Simulating Adsorptive Expansion of Zeolites: Application to Biomass-Derived Solutions in Contact with Silicalite. Langmuir, 2013, $29,4866-4876$.

(24) Sakata, Y.; Furukawa, S.; Kondo, M.; Hirai, K.; Horike, N.; Takashima, Y.; Uehara, H.; Louvain, N.; Meilikhov, M.; Tsuruoka, T.; Isoda, S.; Kosaka, W.; Sakata, O.; Kitagawa, S. Shape-Memory Nanopores Induced in Coordination Frameworks by Crystal Downsizing. Science 2013, 339, 193-196.

(25) Neimark, A. V.; Coudert, F.-X.; Boutin, A.; Fuchs, A. H. Stress-Based Model for the Breathing of Metal-Organic Frameworks. J. Phys. Chem. Lett. 2010, 1, 445-449.

(26) Boutin, A.; Coudert, F.-X.; Springuel-Huet, M.-A.; Neimark, A. V.; Férey, G.; Fuchs, A. H. The Behavior of Flexible MIL-53(Al) upon $\mathrm{CH}_{4}$ and $\mathrm{CO}_{2}$ Adsorption. J. Phys. Chem. C 2010, $114,22237-22244$.

(27) Beurroies, I.; Boulhout, M.; Llewellyn, P. L.; Kuchta, B.; Férey, G.; Serre, C.; Denoyel, R. Using Pressure to Provoke the Structural Transition of Metal-Organic Frameworks. Angew. Chem., Int. Ed. 2010, 49, 7526-7529.

(28) Neimark, A. V.; Coudert, F.-X.; Triguero, C.; Boutin, A.; Fuchs, A. H.; Beurroies, I.; Denoyel, R. Structural Transitions in MIL-53 (Cr): View from Outside and Inside. Langmuir, 2011, 27, 4734-4741.

(29) Bousquet, D.; Coudert, F.-X.; Fossati, A. G. J.; Neimark, A. V.; Fuchs, A. H.; Boutin, A. Adsorption induced transitions in soft porous crystals: An osmotic potential approach to multistability and intermediate structures. J. Chem. Phys. 2013, 138, 174706. 
(30) Sugiyama, H.; Watanabe, S.; Tanaka, H.; Miyahara, M. T. Adsorption-Induced Structural Transition of an Interpenetrated Porous Coordination Polymer: Detailed Exploration of Free Energy Profiles. Langmuir 2012, 28, 5093.

(31) Tan, J. C.; Cheetham, A. K. Mechanical properties of hybrid inorganic-organic framework materials: establishing fundamental structure-property relationships. Chem. Soc. Rev. 2011, 40, 1059-1080.

(32) Tan, J. C.; Civalleri, B.; Lin, C.-C.; Valenzano, L.; Galvelis, R.; Chen, P.-F.; Bennett, T. D.; Mellot-Draznieks, C.; Zicovich-Wilson, C. M.; Cheetham, A. K. Exceptionally Low Shear Modulus in a Prototypical Imidazole-Based Metal-Organic Framework. Phys. Rev. Lett. 2012, 108, 095502.

(33) Ortiz, A. U.; Boutin, A.; Fuchs, A. H.; Coudert, F.-X. Anisotropic Elastic Properties of Flexible Metal-Organic Frameworks: How Soft are Soft Porous Crystals? Phys. Rev. Lett. 2012, 109, 195502.

(34) Ortiz, A. U.; Boutin, A.; Fuchs, A. H.; Coudert, F.-X. Metal-organic frameworks with wine-rack motif: What determines their flexibility and elastic properties? J. Chem. Phys. 2013, $138,174703$.

(35) Ortiz, A. U.; Boutin, A.; Fuchs, A. H.; Coudert, F.-X. Investigating the Pressure-Induced Amorphization of Zeolitic Imidazolate Framework ZIF-8: Mechanical Instability Due to Shear Mode Softening J. Phys. Chem. Lett. 2013, 4, 1861-1865. 
(36) Coasne, B.; Haines, J.; Levelut, C.; Cambon, O.; Santoro, M.; Gorelli, F.; Garbarino, G. Enhanced mechanical strength of zeolites by adsorption of guest molecules. Phys. Chem. Chem. Phys. 2011, 13, 20096-20099.

(37) Triguero, C.; Coudert, F.-X.; Boutin, A.; Fuchs, A. H.; Neimark, A. V. Mechanism of Breathing Transitions in Metal-Organic Frameworks. J. Phys. Chem. Lett. 2012, 2, 2033-2037.

(38) Triguero, C.; Coudert, F.-X.; Boutin, A.; Fuchs, A. H.; Neimark, A. V. Understanding adsorption-induced structural transitions in metal-organic frameworks: From the unit cell to the crystal. J. Chem. Phys. 2012, 137, 184702.

(39) Wang, Z.; Cohen, S. M. Modulating Metal-Organic Frameworks To Breathe: A Postsynthetic Covalent Modification Approach. J. Am. Chem. Soc. 2009, 131, 16675-16677.

(40) Henke, S.; Schneemann, A.; Wütscher, A.; Fischer, R. A. Directing the Breathing Behavior of Pillared-Layered Metal-Organic Frameworks via a Systematic Library of Functionalized Linkers Bearing Flexible Substituents. J. Am. Chem. Soc. 2012, 134, 9464-9474.

(41) Watanabe, S.; Sugiyama, H.; Adachi, H.; Tanaka, H.; Miyahara, M. T. Free energy analysis for adsorption-induced lattice transition of flexible coordination framework. J. Chem. Phys. 2009, 130, 164707.

(42) Kitaura, R.; Seki, K.; Akiyama, G.; Kitagawa, S. Porous Coordination-Polymer Crystals with Gated Channels Specific for Supercritical Gases. J. Chem. Phys. 2013, 138, 054708.

(43) Numaguchi, R.; Tanaka, H.; Watanabe, S.; Miyahara, M. T. Simulation study for adsorption-induced structural transition in stacked-layer porous coordination polymers: Equilibrium and hysteretic adsorption behaviors. Angew. Chem. Int. Ed. 2003, 42, 428-431. 
(44) Kondo, A.; Noguchi, H.; Ohnishi, S.; Kajiro, H.; Tohdoh, A.; Hattori, Y.; Xu, W.-C.;

Tanaka, H.; Kanoh, H; Kaneko, K. Novel Expansion/Shrinkage Modulation of 2D Layered MOF Triggered by Clathrate Formation with $\mathrm{CO}_{2}$ Molecules. Nano Lett. 2006, 6, 2581.

(45) Pera-Titus, M.; Farrusseng, D. Guest-Induced Gate Opening and Breathing Phenomena in Soft Porous Crystals: Building Thermodynamically Consistent Isotherms. J. Phys. Chem. C 2012, 116, 1638-1649.

(46) Ghoufi, A; Maurin, G. Hybrid Monte Carlo Simulations Com- bined with a Phase Mixture Model to Predict the Structural Transitions of a Porous Metal-Organic Framework Material upon Adsorption of Guest Molecules. J. Phys. Chem. C 2010, 114, 6496.

(47) Ghoufi, A.; Maurin, G.; Férey, G. Physics Behind the Guest-Assisted Structural Transitions of a Porous Metal-Organic Framework Material. J. Phys. Chem. Lett. 2010, 1, 28102815.

(48) Yot, P. G.; Ma, Q.; Haines, J.; Yang, Q.; Ghoufi, A.; Devic, T.; Serre, C.; Dmitriev, V.; Férey, G.; Zhong, C.; Maurin, G. Large breathing of the MOF MIL-47( $\left.{ }^{\mathrm{vv}}\right)$ under mechanical pressure: a joint experimental-modelling exploration. Chem. Sci. 2012, 3, 1100-1104.

(49) Ghoufi, A; Subercaze, A.; Ma, Q.; Yot, P. G.; Ke, Y.; Puente-Orench, I.; Devic, T.; Guillerm, V.; Zhong, C.; Serre, C.; Férey, G.; Maurin, G. J. Phys. Chem. C 2012, 116, $13289-13295$.

(50) Zhang, L.; Hu, Z.; Jiang, J. Sorption-Induced Structural Transition of Zeolitic Imidazolate Framework-8: A Hybrid Molecular Simulation Study. J. Am. Chem. Soc. 2013, 135, 3722-3728. 
(51) Zang, J.; Nair, S.; Sholl, D. S. Osmotic ensemble methods for predicting adsorptioninduced structural transitions in nanoporous materials using molecular simulations. J. Chem. Phys. 2011, 134, 184103.

(52) Santander, J. E. Tsapatsis, M.; Auerbach, S. M. Simulating Adsorptive Expansion of Zeolites: Application to Biomass-Derived Solutions in Contact with Silicalite. Langmuir. 2013, $29,4866-4876$.

(53) Brochard, L.; Vandamme, M.; Pellenq, R. J.-M. Poromechanics of microporous media. $J$. Mech. Phys. Solids 2012, 60, 606-622.

(54) Kowalczyk, P.; Ciach, A.; Neimark, A. V. Adsorption-induced deformation of microporous carbons: pore size distribution effect. Langmuir, 2008, 24, 6603-6608.

(55) Yang, K. Lu, X.; Lin, Y.; Neimark, A. V. Effects of coal deformation upon $\mathrm{CO}_{2}$ adsorption and sequestration, J. Geophys. Res. Solid Earth, 2011, 116, B08212.

(56) Yang, K. Lu, X.; Lin, Y.; Neimark, A. V. Deformation of coal induced by methane adsorption at geological conditions, Energy \& Fuel, 2010, 24, 5955-5964.

(57) Gor, G. Y.; Neimark, A. V. Adsorption-Induced Deformation of Mesoporous Solids, Langmuir, 2010, 26, 13021-13027.

(58) Gor, G. Y.; Neimark, A. V. Adsorption-Induced Deformation of Mesoporous Solids: Macroscopic Approach and Density Functional Theory, Langmuir, 2011, 27, 6926-6931. 
(59) Tafipolsky, M.; Schmid, R. Systematic First Principles Parameterization of Force Fields for Metal-Organic Frameworks using a Genetic Algorithm Approach J. Phys. Chem. B 2009, $113,1341-1352$.

(60) Tafipolsky, M.; Amirjalayer, S.; Schmid, R. First-Principles-Derived Force Field for Copper Paddle-Wheel-Based Metal-Organic Frameworks. J. Phys. Chem. C 2010, 114, 1440214409.

(61) McDaniel, J. G.; Yu, K.; Schmidt, J. R. Ab Initio, Physically Motivated Force Fields for CO 2Adsorption in Zeolitic Imidazolate Frameworks. J. Phys. Chem. C 2012, 116, 1892-1903.

(62) Mechanics and Physics of Porous Solids, Coussy, O. John Wiley \& Sons, 2010. 